\title{
The effects of blastocyst morphological score and blastocoele re-expansion speed after warming on pregnancy outcomes
}

\author{
Huiqun Yin, Hong Jiang, Ruibing He, Cunli Wang, Jie Zhu, Yang Li \\ Reproductive Medicine Center, 105th Hospital of PLA, Hefei, China
}

Objective: The aim of this study was to investigate associations between the morphology score of blastocysts and blastocoele re-expansion speed after warming with clinical outcomes, which could assist in making correct and cost-effective decisions regarding the appropriate time to vitrify blastocysts and to transfer vitrified-warmed blastocysts.

Methods: A total of 327 vitrified-warmed two-blastocyst transfer cycles in women 38 years old and younger were included in this retrospective study.

Results: The clinical pregnancy rate (CPR) and implantation rate (IR) of transfers of two good-morphology grade 4 blastocysts vitrified on day 5 (64.1\% and $46.8 \%$, respectively) were significantly higher than the CPR and IR associated with the transfers of two good-morphology grade 3 blastocysts vitrified on day 5 ( $46.7 \%$ and $32.2 \%$, respectively). No significant differences were found in the CPR and IR among the transfers of two good-morphology grade 4 blastocysts regardless of the day of cryopreservation. Logistic regression analysis showed that blastocoele reexpansion speed after warming was associated with the CPR.

Conclusion: The selection of a good-morphology grade 4 blastocyst to be vitrified could be superior to the choice of a grade 3 blastocyst. Extending the culture of grade 3 blastocysts and freezing grade 4 or higher blastocysts on day 6 could lead to a greater likelihood of pregnancy. Since re-expansion was shown to be a morphological marker of superior blastocyst viability, blastocysts that quickly re-expand after warming should be prioritized for transfer.

Keywords: Blastocyst; Embryo transfer; Embryonic development; Freezing; Pregnancy outcome

\section{Introduction}

With refinements in embryo cryopreservation technology in recent years allowing almost all embryos to survive the vitrification/warming process, vitrified-warmed embryo transfer (VET) has become increasingly common. VET is advantageous in that it effectively prevents in vitro fertilization-associated complications such as ovarian

Received: Nov 28, 2015 · Revised: Dec 23, 2015 · Accepted: Jan 21, 2016 Corresponding author: Hong Jiang

Reproductive Medicine Center, 105th Hospital of PLA, 424 West Changjiang Road, Hefei 230031, China

Tel:+86-551-65966361 Fax:+86-551-65123513 E-mail:jiangh105@sina.com

This is an Open Access article distributed under the terms of the Creative Commons Attribution Non-Commercial License (http://creativecommons.org/licenses/by-nc/3.0/) which permits unrestricted non-commercial use, distribution, and reproduction in any medium, provided the original work is properly cited. hyperstimulation syndrome [1], is associated with better obstetric and perinatal outcomes [2], and maximizes the embryo utilization rate and increases the cumulative pregnancy rate $[3,4]$, particularly for slowly developing embryos [5] and patients exhibiting early progesterone elevation [6].

Blastulation of human embryos usually occurs on day 5 after fertilization but may be delayed until day 6 or 7. Previous studies have found that the pregnancy rates for fresh transfers performed on day 6 or later were much lower than those performed on day 5 due to declining endometrial receptivity $[7,8]$. However, transfers of vitrified day 6 blastocysts have been reported by some investigators to have comparable pregnancy rates to the transfers of vitrified day 5 blastocysts $[9,10]$. Other studies found the pregnancy rates to be lower $[11,12]$. A meta-analysis comparing vitrified day 6 and day 5 blasto- 
cysts showed lower pregnancy rates and live birth rates in vitrified day 5 blastocysts. However, when the analysis was restricted to studies comparing day 6 and day 5 blastocysts of similar morphology, no difference was found [13]. The authors suggested that blastocyst expansion extent (regardless of the number of days after fertilization) or blastocyst re-expansion capacity after warming may affect the pregnancy outcomes in vitrified-warmed blastocyst transfer cycles.

Gardner and Schoolcraft [14] set up an easy and noninvasive threeparameter scoring system depending on blastocyst expansion, inner cell mass (ICM), and trophectoderm (TE) development. Several reports have demonstrated that TE morphology is significantly related to pregnancy outcomes [15-17]. However, Goto et al. [18] reported no distinction between the relative importance of the ICM and the TE within the same blastocyst expansion in VET cycles. Post-warming blastocysts have experienced multiple morphological changes in the process of vitrification/warming. First, the blastocyst is subjected to artificial shrinking for blastocoele dehydration before vitrification, then experiences the action of cryoprotectants during cooling, followed by rehydration via the removal of cryoprotectants during warming. The shrinkage and swelling of the blastocoele may result in cell damage or even death. It is then difficult to evaluate blastocyst quality in comparison to a fresh blastocyst. The extended culture of post-warming blastocysts provides a chance to evaluate blastocoele re-expansion, which may take place within 1 to 2 hours after warming [19]. However, little information has been published on correlations between blastocoele re-expansion speed, the morphological score of blastocysts, and the pregnancy outcomes of vitrifiedwarmed blastocyst transfers. Furthermore, no uniform criteria for blastocyst cryopreservation exist, although blastocysts with poorer morphological grades did not qualify for cryopreservation in many studies $[9,20]$.

The objective of this study was to investigate the correlations of blastocyst morphological score and blastocoele re-expansion speed with the clinical outcomes of vitrified-warmed blastocyst transfer cycles. In particular, we compared the clinical outcomes and the percentage of fast blastocoele re-expansion ( $250 \%$ blastocoelic cavity formation during 2 hours after warming) in transfers of good-morphology grade 3 blastocysts with those of grade 4 blastocysts vitrified on day 5 after fertilization, in order to obtain information supporting the selection of appropriate blastocysts for vitrification and transfer.

\section{Methods}

\section{Subjects}

This study was a retrospective analysis of vitrified-warmed twoblastocyst transfer cycles from January 2014 to April 2015 in the re- productive center of the 105th Hospital of PLA. The inclusion criteria were: (1) patients $\leq 38$ years of age; (2) non-obese patients with normal levels of basal follicle-stimulating hormone (FSH) (mean, $7.1 \pm 3.1 \mathrm{IU} / \mathrm{L}$ ) and luteinizing hormone (mean, $5.3 \pm 2.5 \mathrm{IU} / \mathrm{L}$ ); (3) blastocysts obtained from the first oocyte retrieval cycle and cryopreserved by vitrification; and (4) patients who underwent the successful transfer of two blastocysts equal or superior to grade $3 \mathrm{CC} \mathrm{ac-}$ cording to Gardner's criteria [14]. A total of 327 VET cycles satisfied the inclusion criteria. All patients provided written informed consent for the procedures. The study was approved by the Ethics Committee of the 105th Hospital of PLA, Hefei, China (2013085).

Blastocysts were evaluated on the basis of the expansion of the blastocoele in addition to the number and cohesiveness of the ICM and TE, according to Gardner's criteria [14]. A good-morphology blastocyst was defined as having a well-expanded blastocoele (grades 3-6) on day 5 or on day 6 (grades 4-6), a well-defined ICM (subgrades A or B) and a single layer of TE cells surrounding the cavity (subgrades A or B). No changes were made to our laboratory protocols during the study period. According to the blastocyst morphological score and the time of blastocyst freezing (with the day of fertilization termed day 0 ), the transfer cycles were categorized into six groups: BL-1, two good-morphology grade 3 blastocysts frozen on day 5 ; BL-2, two good-morphology grade 4 blastocysts frozen on day 5; BL-3, one good-morphology grade 3 blastocyst and one goodmorphology grade 4 blastocyst, with both frozen on day 5; BL-4, two good-morphology grade 4 blastocysts frozen on day 6 ; BL-5, one good-morphology blastocyst and one poor-quality blastocyst; BL-6, two poor-morphology blastocysts.

Transfer cycles involving two quickly re-expanding blastocysts ( $\geq 50 \%$ blastocoele re-expansion during 2 hours after warming) were defined as the re-expansion group 1. Transfers with one quickly and one slowly re-expanding blastocyst $(<50 \%$ blastocoele re-expansion) were defined as the re-expansion group 2. Those with no quickly re-expanding blastocysts were defined as the re-expansion group 3. Theoretically, blastocysts exhibiting re-expansion or slow reexpansion after warming were considered to have lower development potential. Good-morphology blastocysts could have been prioritized for warming if more than two blastocysts underwent vitrification during the study. Moreover, the study was limited by the fact that the patients in each group were not matched, and that the reexpansion group 3 had a small sample size.

\section{Ovarian stimulation and embryo culture}

Ovarian stimulation was carried out according to standard gonadotropin-releasing hormone agonist/FSH, antagonist/FSH, or ministimulation protocols. Ovulation was triggered by human chorionic gonadotropin when at least three follicles reached $18 \mathrm{~mm}$ in diame- 
ter, followed by oocyte retrieval approximately 36 to 38 hours after human chorionic gonadotropin administration. Oocytes were fertilized by conventional in vitro fertilization or intracytoplasmic sperm injection, according to the results of the semen analysis. The fertilized oocytes were individually cultured in $20-\mu \mathrm{L}$ micro-drops of pre-equilibrated G1-plus medium (Vitrolife, Gothenburg, Sweden) under mineral oil until day 3. The embryos were then transferred into $20-\mu \mathrm{L} \mathrm{mi-}$ cro-drops of pre-equilibrated G2-plus (Vitrolife) until day 5 to 7 . All embryos were incubated at $37^{\circ} \mathrm{C}$ in $6 \% \mathrm{CO}_{2}$.

\section{Blastocyst vitrification freezing and warming procedures}

In this study, only blastocysts equal or superior to grade $3 C \mathrm{C}$ were selected for cryopreservation using a commercial vitrification freezing/warming kit (Kitazato Biopharma Co., Shizuoka, Japan) and all freezing/warming steps were carried out according to the manufacturer's instructions. Briefly, the blastocysts were artificially shrunk for dehydration of the blastocoele by the laser method, and one hole of $10 \mu \mathrm{m}$ on the zona pellucida was made with one or two laser beams at a site far from the ICM using an irradiation time of $3.0 \mathrm{msec}$ (Octax Eyeware, Bruckberg, Germany). After completing the shrinkage of the blastocoele, blastocysts were placed into equilibration solution for 5 minutes at room temperature, then transferred into the vitrification solution for approximately 45 seconds and loaded into the tip of a hemi-straw carrier, with a very small total volume, and were immediately plunged into liquid nitrogen with protection provided by the plastic cover. For the warming procedure, the straw containing the blastocysts was transferred into $1 \mathrm{~mL}$ of thawing solution with $1.0 \mathrm{M}$ sucrose (pre-warmed at $37^{\circ} \mathrm{C}$ ) for 1 minute. Thereafter, the blastocysts were transferred sequentially into dilution solutions with $0.5 \mathrm{M}$ sucrose for 3 minutes and washed twice with washing solution for 5 minutes at room temperature. Post-warming blastocyst survival was defined as $>50 \%$ of the cells remaining intact [19]. Blastocysts were cultured for 1 to 2 hours in order to assess the extent of blastocoele re-expansion, and then were transferred into the uterine cavity.

In our experience, as described in previously published studies [21], high-quality blastocysts started to re-expand within 1 hour after thawing. Blastocysts with a blastocoele re-expansion of more than $50 \%$ of the original size within 2 hours were defined as quickly re-expanding blastocysts, while those with a blastocoele re-expansion of less than $50 \%$ of the original size were regarded as slowly re-expanding blastocysts, and those not showing any sign of re-expansion were classified as non-expanding blastocysts.

\section{Endometrial preparation for VET}

VET was carried out in hormone replacement cycles or in natural cycles. Endometrial preparation was performed on the third day of menstruation in hormone replacement cycles, which comprised ap- proximately $95 \%$ of the cycles. Estradiol valerate tablets (Progynova, Bayer Schering, Guangzhou, China) were given at a dose of 4 to $8 \mathrm{mg}$ per day, with the dose modified according to endometrial thickness and morphology. When the endometrial thickness reached $\geq 8 \mathrm{~mm}$, $60 \mathrm{mg}$ of progestin (progesterone injection, Xianju pharmacy Co., Taizhou, China) was administered intramuscularly once a day for 5 days.

\section{Embryo transfer and pregnancy diagnosis}

Two blastocysts were transferred into the uterine cavity with a Wallace catheter (Wallace, Brisbane, Australia) under abdominal ultrasound guidance. Observation of a gestational sac with a beating fetal heart on day 30 to 35 after embryo transfer was defined as a clinical pregnancy. The number of gestational sacs with a beating fetal heart per number of embryos transferred was defined as the implantation rate (IR). Embryo developmental arrest or spontaneous abortion following less than 12 weeks of gestation was defined as miscarriage.

\section{Statistical analysis}

Comparisons of continuous variables were carried out using oneway analysis of variance when the data were normally distributed. For non-continuous variables, statistical comparisons were carried out using the chi-square test or Fisher's exact test with multiplecomparison tests where appropriate. The interaction of blastocoele re-expansion speed (re-expansion groups 1-3) with clinical outcomes was examined by logistic regression analysis, allocating numerical scores of 1 to 3 for re-expansion groups 1 to 3 . The $p$-values $<0.05$ were considered to indicate statistical significance.

\section{Results}

No significant differences were found among groups BL-1 through BL-6 regarding female age at embryo freezing, the proportion of primary infertility, infertility duration, the intracytoplasmic sperm injection ratio, the cause of infertility, or endometrial thickness (Table 1).

The total clinical pregnancy rate (CPR) of transfers with two goodmorphology blastocysts (BL-1, BL-2, BL-3, and BL-4 groups) was $60.6 \%$ (157/259), which was significantly higher than that of transfers with two poor-morphology blastocysts (BL-6 group, 35.0\%) $(p<0.05)$. The total IR of transfers with two good-morphology blastocysts (BL-1, BL-2, BL-3, and BL-4 groups) was 43.4\% (225/518), which significantly higher than when the transfer involved either one good-morphology blastocyst (BL-5 group, 31.3\%) $(p<0.05)$ or no good-morphology blastocysts (BL-6 group, 20.0\%) $(p<0.01)$.

The CPR and IR of transfers of two good-morphology grade 4 blastocysts frozen on day 5 (BL-2 group: $64.1 \%$ and $46.8 \%$, respectively) were significantly higher than the corresponding values for two 
Table 1. Patients' characteristics of vitrified-warmed blastocysts transfer cycles according to blastocyst quality score (BL-1 to BL-6)

\begin{tabular}{|c|c|c|c|c|c|c|}
\hline Characteristic & BL-1 & BL-2 & BL-3 & $B L-4$ & BL-5 & BL-6 \\
\hline Cycle (n) & 45 & 142 & 56 & 16 & 48 & 20 \\
\hline Age at freezing $(\mathrm{yr})^{\mathrm{a})}$ & $29.8 \pm 3.5$ & $29.6 \pm 3.3$ & $29.3 \pm 3.9$ & $30.4 \pm 4.0$ & $29.5 \pm 3.8$ & $30.1 \pm 4.8$ \\
\hline Primary infertility ${ }^{b}$ & $25(55.6)$ & $74(52.1)$ & $32(57.1)$ & $7(43.8)$ & $26(54.2)$ & $6(30.0)$ \\
\hline Infertility period $(y r)^{a)}$ & $3.9 \pm 2.9$ & $4.3 \pm 2.4$ & $4.1 \pm 3.3$ & $4.0 \pm 2.5$ & $3.7 \pm 2.8$ & $3.8 \pm 1.9$ \\
\hline \multicolumn{7}{|l|}{ Cause of infertility } \\
\hline Endometriosis & $1(2.2)$ & $3(2.1)$ & 0 & 0 & $1(2.1)$ & 0 \\
\hline Ovulation dysfunction & $2(4.4)$ & $7(4.9)$ & $1(1.8)$ & 0 & $1(2.1)$ & 0 \\
\hline Male factor & $5(11.1)$ & $24(16.9)$ & $9(16.1)$ & $3(18.8)$ & $10(20.8)$ & $2(10.0)$ \\
\hline Tubal factor & $32(71.1)$ & $90(63.4)$ & $41(73.2)$ & $11(68.8)$ & $34(70.8)$ & $13(65.0)$ \\
\hline Unexplained & $1(2.3)$ & $2(1.4)$ & $1(1.8)$ & $1(6.2)$ & $1(2.1)$ & $1(5.0)$ \\
\hline Multiple & $4(8.9)$ & $16(11.3)$ & $4(7.1)$ & $1(6.2)$ & $1(2.1)$ & $4(20.0)$ \\
\hline$|\mathrm{ICS}|^{\mathrm{c})}$ & $7(15.6)$ & $35(24.7)$ & $11(19.6)$ & $3(18.8)$ & $10(20.8)$ & $4(20.0)$ \\
\hline Endometrial thickness $(\mathrm{mm})^{\mathrm{a})}$ & $10.2 \pm 1.9$ & $9.8 \pm 1.4$ & $10.1 \pm 1.5$ & $9.9 \pm 1.6$ & $10.0 \pm 1.1$ & $10.1 \pm 1.3$ \\
\hline
\end{tabular}

Value are presented as mean \pm standard deviation or number (\%).

BL-1, two good-morphology blastocyst of grade 3 frozen on day 5; BL-2, two good-morphology blastocyst of grade 4 frozen on day 5; BL-3, one good-morphology blastocyst of grade 3 and the other of grade 4, and both frozen on day 5; BL-4, two good-morphology, blastocyst of grade 4 frozen on day 6; BL-5, one good-morphology blastocyst and one poor-quality blastocyst; BL-6, two poor-morphology blastocysts; ICSI, intracytoplasmic sperm injection.

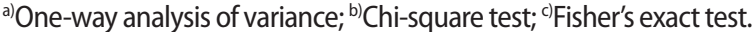

Table 2. Pregnancy outcomes and blastocoele re-expansion of vitrifed-warmed blastocysts transfer cycles according to blastocyst quality score (BL-1 to BL-6)

\begin{tabular}{|c|c|c|c|c|c|c|}
\hline Characteristic & BL-1 & BL-2 & BL-3 & BL-4 & BL-5 & BL-6 \\
\hline Cycle (n) & 45 & 142 & 56 & 16 & 48 & 20 \\
\hline Clinical pregnancy rate ${ }^{\mathrm{a})}$ & $46.7(21 / 45)^{b)}$ & $64.1(91 / 142)$ & $62.5(35 / 56)$ & $62.5(10 / 16)$ & $47.9(23 / 48)^{b)}$ & $35.0(7 / 20)^{b, c}$ \\
\hline Implantation rate ${ }^{\mathrm{a})}$ & $32.2(29 / 90)^{b)}$ & $46.8(133 / 284)$ & $45.5(51 / 112)$ & $37.5(12 / 32)$ & $31.3(30 / 96)^{\mathrm{b}, \mathrm{c}}$ & $20.0(8 / 40)^{b, c)}$ \\
\hline Miscarriage rate ${ }^{\mathrm{d})}$ & $4.8(1 / 21)$ & $12.1(11 / 91)$ & $8.6(3 / 35)$ & $20.0(2 / 10)$ & $21.7(5 / 23)$ & $14.3(1 / 7)$ \\
\hline Multiple pregnancy rate ${ }^{\mathrm{d})}$ & $47.6(10 / 21)$ & $46.2(42 / 91)$ & $48.6(17 / 35)$ & $20.0(2 / 10)$ & $26.1(6 / 23)$ & $14.3(1 / 7)$ \\
\hline Re-expansion $1^{\text {a) }}$ & $66.7(30 / 45)^{b)}$ & $81.7(116 / 142)$ & $76.8(43 / 56)$ & $81.3(13 / 16)$ & $62.5(30 / 48)^{b)}$ & $60.0(12 / 20)^{b)}$ \\
\hline Re-expansion $2^{\mathrm{d})}$ & $28.9(13 / 45)^{b)}$ & $12.7(18 / 142)$ & $16.1(9 / 56)$ & $18.8(3 / 16)$ & $33.3(16 / 48)^{b)}$ & $30.0(6 / 20)^{b)}$ \\
\hline Re-expansion 3 & $4.4(2 / 45)$ & $5.6(8 / 142)$ & $7.1(4 / 56)$ & 0 & $4.2(2 / 48)$ & $10.0(2 / 20)$ \\
\hline
\end{tabular}

Values are presented as percent (number).

BL-1, two good-morphology blastocyst of grade 3 frozen on day 5; BL-2, two good-morphology blastocyst of grade 4 frozen on day 5; BL-3, one good-morphology blastocyst of grade 3 and the other of grade 4, and both frozen on day 5; BL-4, two good-morphology blastocyst of grade 4 frozen on day 6; BL-5, one good-morphology blastocyst and one poor-quality blastocyst; BL-6, two poor-morphology blastocysts; Re-expansion 1, two blastocysts with $\geq 50 \%$ blastocoele re-expansion; Re-expansion 2, one blastocysts with $\geq 50 \%$ blastocoele re-expansion and the other with $<50 \%$ blastocoele re-expansion; Re-expansion 3 , two blastocysts with $<50 \%$ blastocoele re-expansion.

${ }^{\mathrm{a})}$ Chi-square test; ${ }^{\mathrm{b}}$ Compared with $\mathrm{BL}-2, p<0.05 ;{ }^{\mathrm{c}}$ Compared with $\mathrm{BL}-3, p<0.05 ;{ }^{\mathrm{d})}$ Fisher's exact test.

good-morphology grade 3 blastocysts frozen on day 5 (BL-1 group: $46.7 \%$ and $32.2 \%$, respectively), only one good-morphology blastocyst (BL-5 group: $47.9 \%$ and $31.3 \%$, respectively) and two poor-morphology blastocysts (BL-6 group: $35.0 \%$ and $20.0 \%$, respectively) $(p<0.05)$. Moreover, no significant differences were found in the CPR and IR among the transfers of two good-morphology blastocysts involving grade 4, regardless of the day of cryopreservation (BL-2, BL-3, and BL-4 groups). No significant differences were observed in the rates of miscarriage and multiple pregnancies among the six groups (Table 2).

Fast re-expansion was exhibited by $84.6 \%$ of the blastocysts
(553/654) after warming, and $94.5 \%$ of the transfer cycles (309/327) contained at least one quickly re-expanding blastocyst. The CPR and IR in re-expansion group 1 (two quickly re-expanding blastocysts) were significantly higher than in re-expansion group 2 (one quickly and one slowly re-expanding blastocyst) and re-expansion group 3 (no quickly re-expanding blastocysts) (CPR: $62.3 \%$ vs. $44.6 \%$ vs. $33.3 \%, p<0.05$; IR: $44.3 \%$ vs. $30.8 \%$ vs. $19.4 \%, p<0.01)$. Logistic regression analysis also showed that blastocoele re-expansion speed after warming was associated with the CPR (odds ratio, 0.56; 95\% confidence interval, 0.35-0.78), with numerical scores of 1 to 3 allocated for re-expansion groups 1 through 3 . No statistically significant 
Table 3. Pregnancy outcomes of vitrified-warmed blastocyst transfer cycles according to blastocoele re-expansion speed after warming

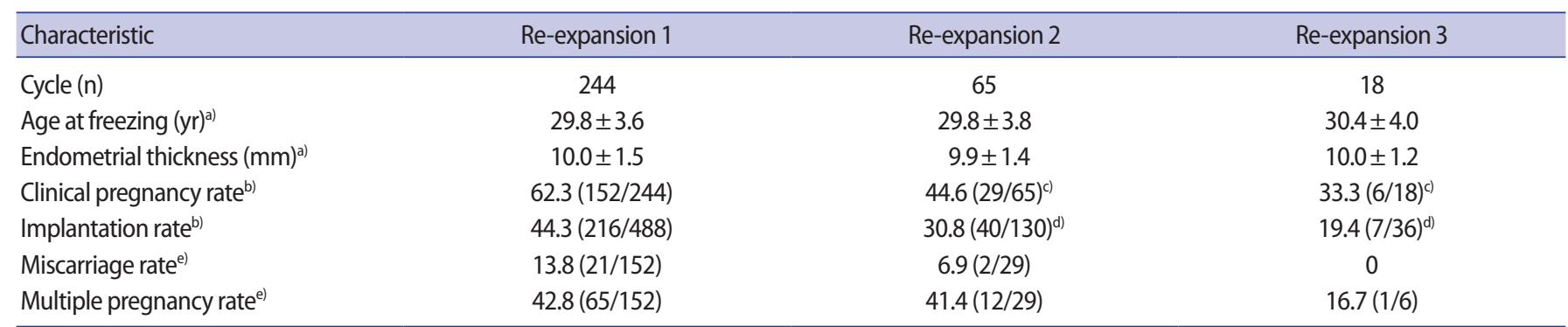

Values are presented as mean \pm standard deviation or percent (number).

Re-expansion 1, two blastocysts with $\geq 50 \%$ blastocoele re-expansion; Re-expansion 2, one blastocysts with $\geq 50 \%$ blastocoele re-expansion and the other with $<50 \%$ blastocoele re-expansion; Re-expansion 3, two blastocysts with $<50 \%$ blastocoele re-expansion.

a) One-way analysis of variance; ${ }^{\text {b) }}$ Chi-square test; ${ }^{\text {c) }} p<0.05$; ${ }^{\mathrm{d}} p<0.01$; ${ }^{\text {e) }}$ Fisher's exact test; compared with re-expansion 1.

differences were found in the rates of miscarriage and multiple pregnancies among the three groups (Table 3). In addition, 6.1\% (40/654) of vitrified-warmed blastocysts did not re-expand within 2 hours, and one ongoing clinical pregnancy was established from the 10 blastocysts without blastocoele re-expansion that were transferred in re-expansion group 3.

The percentage of cycles involving two quickly re-expanding blastocysts in the BL-2 group (good-morphology grade 4 blastocysts) was higher than in the BL-1 group (good-morphology grade 3 blastocysts) $(p<0.05)$, which was consistent with the clinical pregnancy rates (Table 2, Figure 1).

\section{Discussion}

The results of our study showed that transferring two good-morphology grade 4 ( $4 \mathrm{AA} / 4 \mathrm{AB} / 4 \mathrm{BA} / 4 \mathrm{BB})$ blastocysts frozen on day 5 achieved a higher CPR and IR than was obtained when transferring grade $3(3 \mathrm{AA} / 3 \mathrm{AB} / 3 \mathrm{BA} / 3 \mathrm{BB})$ blastocysts frozen on day 5 and either one or no good-morphology blastocysts. No significant difference was found in the CPR or IR when two good-morphology grade 4 blastocysts were transferred, regardless of the day of cryopreservation. Logistic regression analysis showed that blastocoele re-expansion speed after warming was associated with the CPR.

Previous studies have evaluated the influence of blastocyst morphology on pregnancy rates. Gardner et al. [22] reported that the blastocyst morphological score had a predictive value for embryo development competence, and a significantly higher CPR was achieved when two top-scoring blastocysts were transferred on day 5 , in comparison with the transfers of either one top-scoring blastocyst or no top-scoring blastocysts. Veleva et al. [23] analyzed factors affecting the outcome of VET cycles, showing that transfer of the embryo with the highest quality and viability after warming led to better clinical outcomes. Our study confirmed these findings, showing that when two good-morphology blastocysts were transferred, the

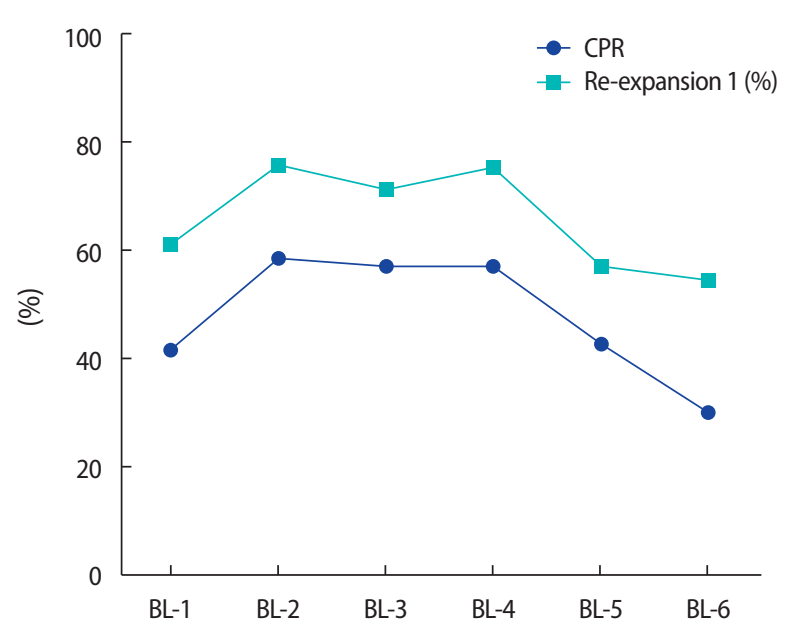

Figure 1. The relationship of the blastocyst morphological score with the blastocoele re-expansion speed after warming and the CPR. BL1, two good-morphology grade 3 blastocysts frozen on day 5; BL-2, two good-morphology grade 4 blastocysts frozen on day 5; BL-3, one good-morphology grade 3 blastocyst and one good-morphology grade 4 blastocyst, with both frozen on day 5; BL-4, two good-morphology grade 4 blastocysts frozen on day $6 ; B L-5$, one good-morphology blastocyst and one poor-quality blastocyst; BL-6, two poormorphology blastocysts; CPR, clinical pregnancy rate; re-expansion 1 (\%), the percentage of cycles involving two quickly re-expanding blastocysts.

CPR and IR were higher than when either one good-morphology blastocyst or no good-morphology blastocysts were transferred.

In a study of 1,488 single frozen-thawed blastocyst transfer cycles, Goto et al. [18] found a significant correlation between blastocyst quality and the CPR. However, neither ICM nor TE affected the pregnancy outcomes within the same grade 3 or grade 4 blastocysts. In the present study, the transfer of two good-quality grade 4 blastocysts frozen on day 5 achieved a higher CPR and IR than was achieved when two good-quality grade 3 blastocysts frozen on day 5 were transferred, suggesting that the blastocyst grade (morphology and 
blastocyst expansion extent) before freezing affected pregnancy outcomes in vitrified-warmed blastocyst transfer cycles. This result was similar to those reported by Goto et al. [18], who showed that the CPR associated with the transfer of grade $3(3 \mathrm{AA} / 3 \mathrm{AB} / 3 \mathrm{BB})$ blastocysts was $49.17 \%$, which was lower than that of grades $6,5,4 A A$, $4 A B$, and $4 B A(67.09 \%)$ in patients 38 years old and younger. These results suggest that embryo development to the expanded blastocyst stage (higher than grade 4) is an important parameter for pregnancy outcomes.

More cycles in which two quickly re-expanding good-morphology blastocysts were transferred involved grade 4 blastocysts than grade 3 blastocysts, while similar CPRs were observed. Transfer cycles containing at least one quickly re-expanding blastocyst achieved an approximately two-fold higher CPR and IR than those without quickly re-expanding blastocysts, which was consistent with the results of Shu et al. [24]. This suggests that the extended culture of early blastocysts to the expanded blastocyst stage for freezing may enhance their ability to tolerate cryopreservation agents and facilitate blastocyst survival and re-expansion after warming. In addition, goodmorphology grade 4 blastocysts had a greater number of TE cells, better compaction of the ICM, and a larger blastocoele volume, resulting in thinning of the zona pellucida, which could facilitate implantation in vitrified-warmed blastocyst transfer cycles in comparison with good-morphology grade 3 blastocysts. Previous studies have revealed that extending the culture of post-warming expanded blastocysts and transferring spontaneously hatching/hatched blastocysts can result in higher implantation and pregnancy rates [5]. Therefore, the selection of expanded grade 4 blastocysts to be frozen on day 5 or extending the culture of post-thaw grade 3 or 4 blastocysts to the hatching/hatched stage for transfer may lead to better pregnancy outcomes.

In addition, Hashimoto et al. [12] reported that IR of vitrifiedwarmed blastocysts frozen on day 5 (normally developing embryos) was higher than that of those that required culture to day 6 , and the incidence of abnormal spindles in the growth-retarded blastocysts was higher than that in normally developing blastocysts. However, in the present study, no significant difference was found in the CPR or IR among the transfers of two good-morphology grade 4 blastocysts regardless of the day of cryopreservation. This difference may have been due to the small sample (16 cycles) of transfers of good-morphology grade 4 blastocysts frozen on day 6 .

Blastocysts that did not show significant re-expansion after warming were more susceptible to the procedure of vitrification freezing and warming, and had a lower survival rate than expanded blastocysts [19]. However, it was noteworthy that one ongoing clinical pregnancy was established from 10 transferred blastocysts that did not exhibit blastocoele re-expansion 2 hours post-warm culture, sug- gesting that unexpanded blastocysts 2 hours post-warm culture still had some likelihood of implanting on the endometrium. These results agree with those reported in other studies, which showed that some shrunken post-warm blastocysts could develop to the hatched stage after thawing for 5 to 6 hours and that the shrunken blastocyst exhibited sufficient implantation ability [25].

In conclusion, the total IR of transfers with two good-morphology blastocysts was significantly higher than that observed with either one good-morphology blastocyst or no good-morphology blastocysts. Transferring two good-morphology grade 4 blastocysts achieved a higher CPR and IR than was obtained using two goodmorphology grade 3 blastocysts. Simultaneously, more transfers involved two quickly re-expanding grade 4 blastocysts than involved quickly re-expanding grade 3 blastocysts. No significant difference in the CPR or IR was observed among the transfers of two good-morphology grade 4 blastocysts. The speed of blastocoele re-expansion, as a superior morphological marker of embryo viability, was associated with pregnancy outcomes. Therefore, selection of a good-morphology grade 4 blastocyst to be frozen on day 5 would be superior to choosing a grade 3 blastocyst, and extending the post-warming culture grade 3 blastocysts to the hatching/hatched stage for transfer may achieve better pregnancy outcomes. If no re-expanded blastocyst is observed 2 hours post-warm culture, the culture time should be extended to 5 hours. If no blastocoele formation occurs nonetheless, additional warming or cancellation of the blastocyst transfer is a judicious choice.

\section{Conflict of interest}

No potential conflict of interest relevant to this article was reported.

\section{References}

1. Maheshwari A, Bhattacharya S. Elective frozen replacement cycles for all: ready for prime time? Hum Reprod 2013;28:6-9.

2. Maheshwari A, Pandey S, Shetty A, Hamilton M, Bhattacharya S. Obstetric and perinatal outcomes in singleton pregnancies resulting from the transfer of frozen thawed versus fresh embryos generated through in vitro fertilization treatment: a systematic review and meta-analysis. Fertil Steril 2012;98:368-77.e1-9.

3. Shen C, Shu D, Zhao X, Gao Y. Comparison of clinical outcomes between fresh embryo transfers and frozen-thawed embryo transfers. Iran J Reprod Med 2014;12:409-14.

4. Wu K, Zhao H, Liu H, Li M, Ma S, Li C, et al. Day 3 ET, single blastocyst transfer (SBT) or frozen-thawed embryo transfer (FET): which is preferable for high responder patients in IVF/ICSI cycles? J Assist Reprod Genet 2014;31:275-8. 
5. Chimote NM, Chimote NN, Nath NM, Mehta BN. Transfer of spontaneously hatching or hatched blastocyst yields better pregnancy rates than expanded blastocyst transfer. J Hum Reprod Sci 2013;6:183-8.

6. Shapiro BS, Daneshmand ST, Garner FC, Aguirre M, Hudson C, Thomas S. Embryo cryopreservation rescues cycles with premature luteinization. Fertil Steril 2010;93:636-41.

7. Shapiro BS, Richter KS, Harris DC, Daneshmand ST. A comparison of day 5 and day 6 blastocyst transfers. Fertil Steril 2001;75:112630.

8. Barrenetxea G, Lopez de Larruzea A, Ganzabal T, Jimenez R, Carbonero K, Mandiola M. Blastocyst culture after repeated failure of cleavage-stage embryo transfers: a comparison of day 5 and day 6 transfers. Fertil Steril 2005;83:49-53.

9. El-Toukhy T, Wharf E, Walavalkar R, Singh A, Bolton V, Khalaf Y, et al. Delayed blastocyst development does not influence the outcome of frozen-thawed transfer cycles. BJOG 2011;118:1551-6.

10. Liebermann J, Tucker MJ. Comparison of vitrification and conventional cryopreservation of day 5 and day 6 blastocysts during clinical application. Fertil Steril 2006;86:20-6.

11. Levens ED, Whitcomb BW, Hennessy S, James AN, Yauger BJ, Larsen FW. Blastocyst development rate impacts outcome in cryopreserved blastocyst transfer cycles. Fertil Steril 2008;90: 2138-43.

12. Hashimoto S, Amo A, Hama S, Ito K, Nakaoka Y, Morimoto Y. Growth retardation in human blastocysts increases the incidence of abnormal spindles and decreases implantation potential after vitrification. Hum Reprod 2013;28:1528-35.

13. Sunkara SK, Siozos A, Bolton VN, KhalafY, Braude PR, El-Toukhy T. The influence of delayed blastocyst formation on the outcome of frozen-thawed blastocyst transfer: a systematic review and meta-analysis. Hum Reprod 2010;25:1906-15.

14. Gardner DK, Schoolcraft WB. Culture and transfer of human blastocysts. Curr Opin Obstet Gynecol 1999;11:307-11.

15. Ahlstrom A, Westin C, Reismer E, Wikland M, Hardarson T. Trophectoderm morphology: an important parameter for predicting live birth after single blastocyst transfer. Hum Reprod 2011;26: 3289-96.
16. Chen X, Zhang J, Wu X, Cao S, Zhou L, Wang Y, et al. Trophectoderm morphology predicts outcomes of pregnancy in vitrifiedwarmed single-blastocyst transfer cycle in a Chinese population. J Assist Reprod Genet 2014;31:1475-81.

17. Honnma H, Baba T, Sasaki M, Hashiba Y, Ohno H, Fukunaga T, et al. Trophectoderm morphology significantly affects the rates of ongoing pregnancy and miscarriage in frozen-thawed singleblastocyst transfer cycle in vitro fertilization. Fertil Steril 2012; 98:361-7.

18. Goto S, Kadowaki T, Tanaka S, Hashimoto H, Kokeguchi S, Shiotani M. Prediction of pregnancy rate by blastocyst morphological score and age, based on 1,488 single frozen-thawed blastocyst transfer cycles. Fertil Steril 2011;95:948-52.

19. Kosasa TS, McNamee PI, Morton C, Huang TT. Pregnancy rates after transfer of cryopreserved blastocysts cultured in a sequential media. Am J Obstet Gynecol 2005;192:2035-9.

20. Van den Abbeel E, Camus M, Verheyen G, Van Waesberghe L, Devroey $P$, Van Steirteghem A. Slow controlled-rate freezing of sequentially cultured human blastocysts: an evaluation of two freezing strategies. Hum Reprod 2005;20:2939-45.

21. Berin I, McLellan ST, Macklin EA, Toth TL, Wright DL. Frozenthawed embryo transfer cycles: clinical outcomes of single and double blastocyst transfers. J Assist Reprod Genet 2011;28:57581.

22. Gardner DK, Lane M, Stevens J, Schlenker T, Schoolcraft WB. Blastocyst score affects implantation and pregnancy outcome: towards a single blastocyst transfer. Fertil Steril 2000;73:1155-8.

23. Veleva Z, Orava M, Nuojua-Huttunen S, Tapanainen JS, Martikainen $\mathrm{H}$. Factors affecting the outcome of frozen-thawed embryo transfer. Hum Reprod 2013;28:2425-31.

24. Shu Y, Watt J, Gebhardt J, Dasig J, Appling J, Behr B. The value of fast blastocoele re-expansion in the selection of a viable thawed blastocyst for transfer. Fertil Steril 2009;91:401-6.

25. Maezawa T, Yamanaka M, Hashimoto S, Amo A, Ohgaki A, Nakaoka Y, et al. Possible selection of viable human blastocysts after vitrification by monitoring morphological changes. J Assist Reprod Genet 2014;31:1099-104. 